\title{
NORMALS, SUBNORMALS AND AN OPEN QUESTION
}

\author{
FRANCISZEK HugON SZAFRANIEC
}

Abstract. An acute look at basic facts concerning unbounded subnormal operators is taken here. These operators have the richest structure and are the most exciting among the whole family of beneficiaries of the normal ones. Therefore, the latter must necessarily be taken into account as the reference point for any exposition of subnormality. So as to make the presentation more appealing a kind of comparative survey of the bounded and unbounded case has been set forth.

This piece of writing serves rather as a practical guide to this largely impenetrable territory than an exhausting report. 44A60.

Mathematics subject classification (2010): Primary 47A20, 47B15, 47B20, 47B25; Secondary 43A35,

Keywords and phrases: Bounded operator, unbounded operator, normal operator, $*$-cyclic operator, cyclic operator, quasinormal operator, subnormal operator, minimality of normal extension, minimality of spectral type, semispectral measure, elementary spectral measure, minimality of cyclic type, uniqueness of extensions, invariant domain, complex moment problem, determinacy of measure, ultradeterminacy of measure, vector of determinacy, vector of ultradeterminacy, creation operator, quantum harmonic oscillator, Segal-Bargmann space, analytic model, reproducing kernel Hilbert space, integrability of RKHS.

\section{REFERENCES}

[1] T. Andô, Matrices of normal extensions of subnormal operators, Acta Sci. Math. (Szeged), 24 (1963), 91-96.

[2] V. Bargmann, On a Hilbert space of analytic functions and an associated integral transform, Comm. Pure Appl. Math., 14 (1961), 187-214.

[3] G. Biriuk And E. A. Coddington, Normal extensions of unbounded formally normal operators. J. Math. Mech. 13 (1964), 617-637.

[4] M. S. Birman AND M. Z. Solomjak, Spectral theory of self-adjoint operators in Hilbert space, D. Reidel Publishing Company, Dordrecht, Boston, Lancaster, Tokyo, 1987.

[5] E. Bishop, Spectral theory of operators on a Banach space, Trans. Amer. Math. Soc., 86 (1957), 414-445.

[6] J. BRAM, Subnormal operators, Duke Math. J, 22 (1955), 75-94.

[7] A. BRown, On a class of operators, Proc. Amer. Math. Soc., 4 (1953), 723-728.

[8] D. CichoŃ, Jan Stochel and F.H. Szafraniec, How much indeterminacy may fit in a moment problem. An example, submitted.

[9] E. A. Coddington, Formally normal operators having no normal extension, Canad. J. Math., 17 (1965), 1030-1040.

[10] Extension theory of formally normal and symmetric subspaces, Memoirs of the American Mathematical Society, No. 134. American Mathematical Society, Providence, R.I., 1973.

[11] J. B. Conway, The theory of subnormal operators, Mathematical Surveys and Monographs, Providence, Rhode Island, 1991.

[12] J. Dieudonné, Treatise on analysis vol. II, Academic Press, New York and London, 1970.

[13] S. L. L. VAn Eijndhoven and J. L. H. Meyers, New orthogonality relations for the Hermite polynomials and related Hilbert spaces, J. Math. Ann. Appl., 146 (1990), 89-98.

[14] C. FoIAŞ, Décomposition en opérateurs et vecteurs propres. I. Études de ces décompositions et leurs rapports avec les prolongements des opérateurs, Rev. Roumaine Math. Pures Appl., 7 (1962), 241-282. 
[15] J. Friedrich, A note on the two dimensional moment problem, Math. Nach, 121 (1985), 285-286.

[16] B. Fuglede, The multidimensional moment problem, Expo. Math., 1 (1983), 47-65.

[17] B. C. Hall, Holomorphic methods in analysis and mathematical physics, Contemp. Math., 260 (2000), 1-59.

[18] P. Halmos, Normal dilations and extensions of operators, Summa Brasiliensis Math., 2 (1950), 125134.

[19] S. Hassi, H. S. V. De Snoo and F. H. Szafraniec, Componentwise decompositions and Cartesian decompositions of linear relations, Dissertationes Math., 465 (2009), 59 pp.

[20] A. S. Holevo, Probabilistic and statistical aspects of quantum theory, North-Holland, Amsterdam New York - Oxford, 1982.

[21] A. Lambert, Subnormality and weighted shifts, J. London Math. Soc., 14 (1976), 476-480.

[22] W. Majdak, Z. Sebestyén, J. Stochel and J. E. Thomson, A local lifting theorem for subnormal operators, Proc. Amer. Math. Soc. 134 (2006), 1687-1699.

[23] W. MAJDAK, A lifting theorem for unbounded quasinormal operators, J. Math. Anal. Appl. 332 (2007), 934-946.

[24] M. A. Naimark, On a representation of additive set functions, C. R. Doklady Acad. Sci. URSS, 41 (1943), 359-361.

[25] E. Nelson, Analytic vectors, Ann. Math. 70 (1959), 572-615.

[26] A. E. Nussbaum, Quasi-analytic vectors, Ark. Mat. 6 (1965), 179-191.

[27] G. MCDonald And C. SundberG, On the spectra of unbounded subnormal operators, Can. J. Math., 38 (1986), 1135-1148.

[28] S. ÔTA, Closed linear operators with domain containing their range, Proc. Edinburgh Math. Soc., 27 (1984), 229-233, see also the MathSciNet review \# MR760619 (86e:47002).

[29] - On normal extensions of unbounded operators, Bulletin of the Polish Acad. Sci. Math., 46 (1998), 291-301.

[30] I. E. Segal, Mathematical problems of relativistic physiscs, Chap. IV in 'Proceedings of the Summer Seminar', Boulder, Colorado 1960, vol II, ed. M. Kac; Lectures in Applied Mathematics, AMS, Providence, RI, 1963.

[31] J. A. Shohat And J. D. Tamarkin, The problem of moments, Amer. Math. Soc. Colloq. Publ., vol. 15, Amer. Math. Soc., Providence, R.I., 1943

[32] J. STOCHEL, Lifting strong commutants of unbounded subnormal operators, Integral Equations Operator Theory, $\mathbf{4 3}$ (2002), 189-214.

[33] J. Stochel AND F. H. SZAFraniec, A characterization of subnormal operators, Operator Theory: Advances and Applications, 14 (1984), 261-263.

[34] - On normal extensions of unbounded operators. I, J. Operator Theory, 14 (1985), 31-55.

[35] - On normal extensions of unbounded operators. II, Acta Sci. Math. (Szeged), 53 (1989), 153177.

[36] - On normal extensions of unbounded operators. III. Spectral properties, Publ. RIMS, Kyoto Univ., 25 (1989), 105-139.

[37] - The normal part of an unbounded operator, Nederl. Akad. Wetensch. Proc. Ser. A, 92 (1989), 495-503 = Indag. Math, 51 (1989), 495-503.

[38] - A few assorted questions about unbounded subnormal operators, Univ. Iagel. Acta Math., 28 (1991), 163-170.

[39] - The complex moment problem and subnormality: a polar decomposition approach, J. Funct. Anal., 159 (1998), 432-491.

[40] - A peculiarity of the creation operator, Glasgow Math. J., 44 (2001), 137-147.

[41] - Unbounded operators and subnormality, monograph in preparation.

[42] F. H. Szafraniec, Dilations on involution semigroups, Proc. Amer. Math. Soc., 66 (1977), 30-32.

[43] - A RKHS of entire functions and its multiplication operator. An explicit example, Operator Theory: Advances and Applications, 43, 309-312, 1990.

[44] - Sesquilinear selection of elementary spectral measures and subnormality, in Elementary Operators and Applications, Proceedings, Blaubeuren bei Ulm (Deutschland), June 9-12, 1991, ed. M.Mathieu, pp. 243-248, World Scientific, Singapore, 1992.

[45] — The Sz.-Nagy "théorème principal" extended. Application to subnormality, Acta Sci. Math. (Szeged), 57 (1993), 249-262. 
[46] - A (little) step towards orthogonality of analytic polynomials, J. Comput. Appl. Math., 49 (1993), 255-261.

[47] - Yet another face of the creation operator, Operator Theory: Advances and Applications, 80, pp. 266-275, Birkhäuser, Basel, 1995.

[48] - Analytic models of the quantum harmonic oscillator, Contemp. Math., 212 (1998), 269-276.

[49] Subnormality in the quantum harmonic oscillator, Commun. Math. Phys., 210 (2000), $323-$ 334.

[50] - The reproducing kernel Hilbert space and its multiplication operators, in Operator Theory: Theory and Applications, vol. 114, pp. 253-263, Birkhäuser, Basel, 2000.

[51] Charlier polynomials and translational invariance in the quantum harmonic oscillator, Math. Nachr., 241 (2002), 163-169.

[52] - Multipliers in the reproducing kernel Hilbert space, subnormality and noncommutative complex analysis, Oper. Theory Adv. Appl., 143 (2003), 313-331.

[53] - How to recognize the creation operator, Rep. Math. Phys., 59 (2007), 401-408.

[54] On normal extensions of unbounded operators. IV. A matrix construction, Oper. Th. Adv. Appl., 163, 337-350, 2005.

[55] - Subnormality and cyclicity, Banach Center Publications, 67 (2005), 349-356.

[56] B. Sz.-NAGY, Spektraldarstellung linearer Transformationen des Hilbertschen Raumes, Springer Verlag, Berlin, 1942.

[57] - Extensions of linear transformations in Hilbert space which extend beyond this space, Appendix to F. Riesz, B. Sz.-Nagy, Functional Analysis, Ungar, New York, 1960

[58] - Unitary dilations of Hilbert space operators and related topics, Appendix no. 2 to F. Riesz, B. Sz.-Nagy, Functional Analysis 2nd Russian edition (extended), Izdat "Mir", Moscow 1979.

[59] J. Weidmann, Linear operators in Hilbert spaces, Springer-Verlag, Berlin, Heidelberg, New York, 1980.

[60] T. T. Trent, New conditions for subnormality, Pacific J. Math., 93 (1981), 459-464.

[61] http://library.thinkquest.org/C0111578/nsindex.html 